\title{
Consumption of combined fructose and sucrose diet exacerbates oxidative stress, hypertrophy and CaMKII $\delta$ oxidation in hearts from rats with metabolic syndrome
}

\section{David Julian Arias-Chávez}

Centro de Investigacion y de Estudios Avanzados del IPN: Centro de Investigacion y de Estudios Avanzados del Instituto Politecnico Nacional

\section{Patrick Mailloux-Salinas}

Centro de Investigacion y de Estudios Avanzados del IPN: Centro de Investigacion y de Estudios Avanzados del Instituto Politecnico Nacional

\section{Julio Altamirano}

Tecnologico de Monterrey: Instituto Tecnologico y de Estudios Superiores de Monterrey

\section{Fengyang Huang}

Hospital Infantil de México Federico Gomez: Hospital Infantil de Mexico Federico Gomez

\section{Norma Leticia Gómez-Viquez}

Centro de Investigacion y de Estudios Avanzados del IPN: Centro de Investigacion y de Estudios Avanzados del Instituto Politecnico Nacional

Guadalupe Bravo ( $\square$ gbravof@yahoo.com )

Centro de Investigación y de Estudios Avanzados del Instituto Politécnico Nacional Departamento de Farmacobiología Sede Sur https://orcid.org/0000-0002-2730-7761

\section{Research Article}

Keywords: metabolic syndrome, cardiac hypertrophy, oxidative stress, CaMKIII high sucrose diet, high fructose diet

Posted Date: September 10th, 2021

DOl: https://doi.org/10.21203/rs.3.rs-876661/v1

License: (c) (1) This work is licensed under a Creative Commons Attribution 4.0 International License. Read Full License 
Version of Record: A version of this preprint was published at Molecular and Cellular Biochemistry on February 9th, 2022. See the published version at https://doi.org/10.1007/s11010-022-04364-w. 


\section{Abstract}

The prevalence of the metabolic syndrome (MetS) and its cardiac comorbidities as cardiac hypertrophy (CH) have increased considerably due to the high consumption of carbohydrates, such as sucrose and/or fructose. We compared the effects of sucrose (S), fructose (F) and their combination (S +F) on the development of MetS in weaned male Wistar rats and established the relationship between the consumption of these sugars and the degree of cardiac $\mathrm{CH}$ development, oxidative stress (OS) and Calcium/calmodulin-dependent protein kinase type II subunit delta oxidation (ox-CaMKII $\delta$ ). 12 weeks after the beginning of treatments with S, F or S +F, arterial pressure (AP) was measured and 8 weeks later (to complete 20 weeks) the animals were sacrificed and blood samples, visceral adipose tissue and hearts were obtained. Biochemical parameters were determined in serum and cardiac tissue to evaluate the development of MetS and OS. To evaluate $\mathrm{CH}$, atrial natriuretic peptide (ANP), CaMKII $\delta$ and ox-CaMKII $\delta$ were determined by western blot and histological studies were performed in cardiac tissue. Our data showed that chronic consumption of $\mathrm{S}+\mathrm{F}$ exacerbates MetS-induced $\mathrm{CH}$ which is related with a higher OS and ox-CaMKII $\delta$.

\section{Introduction}

Free sugars are monosaccharides or disaccharides that have been added to beverages or foods during their preparation or transformation by the food industry [1]. Sucrose (S), obtained from sugarcane or beetroot, and fructose (F), monosaccharide obtained in greater quantity from high fructose corn syrup (HFCS), are the two free sugars used par excellence for the preparation of sweetened beverages [1, 2]. It is estimated that the per capita daily supply of caloric sweeteners is $158 \mathrm{~g}$, where $86 \mathrm{~g}$ are S, $50 \mathrm{~g} \mathrm{HFCS}$ and $22 \mathrm{~g}$ honey and other syrups [1]. Currently, there is an indiscriminate consumption of sugar-sweetened beverages from early ages that exacerbate metabolic problems, and thus complications such as cardiovascular diseases in adulthood. On the other hand, usually, the health implications have been studied when $S$ and $F$ are consumed individually and not in combination [3-6]. Therefore, little is known about the possible metabolic damage and other complications that may be caused by their combined consumption from an early age, mimicking the current trend of human consumption of these sugars.

Excess intake of free sugars increases the accumulation of adipose tissue, which, together with sedentary lifestyles, have contributed significantly to the long-term development of metabolic syndrome (MetS) [7, 8]. MetS has become a pandemic and a global public health problem due to its high prevalence in recent years [7-9]. MetS is characterized by a set of metabolic disorders including dyslipidemias, increased arterial pressure (AP) or overt arterial hypertension (AH), hyperglycemia, hyperuricemia, insulin resistance (IR), oxidative stress (OS) and obesity, the latter disorder being a key factor in the etiology of the syndrome $[8,10]$. MetS is a risk factor for developing type 2 diabetes mellitus (DM2), hepatic steatosis, cardiovascular disease (CVD) and premature mortality. MetS pathogenesis is very complex, and many details are still unclear, but it has been observed that these metabolic disorders are occurring at increasingly younger ages [7-9]. 
It has been reported that excess visceral adipose tissue in patients with MetS causes an increase in the production of reactive oxygen species (ROS) and a decrease in the antioxidant enzymes, leading to OS. [9]. In the heart, OS contributes to development of prediabetic and diabetic heart disease [11] by promoting irreversible protein oxidation, mitochondrial and cellular damage and even cell death. There are several cardiac proteins functionally sensitive to pro-oxidant conditions. In this sense, it has been reported that $\mathrm{Ca}^{2+} /$ calmodulin-dependent protein kinase II (CaMKII) can undergo oxidative postraductional modifications. CaMKII is a serine/threonine kinase that requires the $\mathrm{Ca}^{2+} /$ calmodulin $\left(\mathrm{Ca}^{2+} / \mathrm{CaM}\right)$ complex to be activated. In the heart, the predominant isoform is CaMKII $\delta$, which participates in the regulation of membrane excitability, cellular $\mathrm{Ca}^{2+}$ homeostasis, metabolism and gene transcription [12]. Nonetheless, during its activation, ATP-mediated autophosphorylation of threonine 287 on the regulatory site can take place, which allows $\mathrm{CaMKII}_{\delta}$ to remain chronically active and independent of $\mathrm{Ca}^{2+} /$ CaM complex, triggering different pathological responses $[12,13]$. Similarly, it has been reported that an increase in ROS production favors oxidation of methionine residues 281 and 282 localized in the regulatory domain of $\mathrm{CaMKII}_{\delta}[12,13]$ Oxidation of $\mathrm{CaMKII}_{\delta}\left(\mathrm{ox}-\mathrm{CaMKII}_{\delta}\right.$ ) leads to chronic activation of this enzyme and contributes to different pathological outcomes such as arrhythmias, apoptosis, and inflammation [11-16]. Moreover, Zhong et al., and other groups have demonstrated that pathological activation of $\mathrm{CaMKII}_{\delta}$ under OS conditions contributes to development of cardiac hypertrophy $(\mathrm{CH})[6$, 16-18]. Nevertheless, the direct relationship between MetS, OS, ox-CaMKII $\delta$ and $\mathrm{CH}$ induced by consumption of sugar-sweetened beverages has not been established.

Accordingly, we decided to evaluate the effects of $S, F$, and S + F consumption in weaned rats on the development of MetS and to establish their relationship with the development of $\mathrm{CH}$ and the levels of ox$\mathrm{CaMKII}_{\delta}$ and OS.

\section{Material And Methods}

Experimental animals. Forty-eight male Wistar rats, freshly weaned (21 days old) from the Cinvestav-IPN Pharmacobiology Department's animal facility were used. The rats were housed under a normal $12 \mathrm{~h}$ light-dark cycle and controlled temperature-humidity $\left(22 \pm 2^{\circ} \mathrm{C}\right.$ and $60 \%$, respectively). The rats were randomized into four $(n=12)$ groups as follows: Control group (C) with normal diet, Sucrose group $(S)$ with sucrose diet, Fructose group (F) with fructose diet, and Sucrose + Fructose Group (S +F) with sucrose and fructose diet. All animal procedures and the protocols of the present investigation were approved by our Institutional Ethics Committee (CICUAL-Cinvestav-IPN) and followed the regulations established by the Mexican Official Norm for the Use and Welfare of Laboratory Animals (NOM-062-ZOO-1999).

Metabolic syndrome induction. All animals received LabDiet 5008® rat chow (Richmond, IN, USA) ad libitum, providing $24.4 \mathrm{~kJ} / \mathrm{g}$, with $23 \%$ protein, $6.5 \%$ fat, $4 \%$ fiber, and $8 \%$ ashes. During a 20 -week period, $\mathrm{C}$ group, were given purified water ad libitum, while $\mathrm{S}$ group received $30 \%(\mathrm{w} / \mathrm{v})$ commercially refined sucrose, $\mathrm{F}$ group received $10 \%$ fructose $(\mathrm{w} / \mathrm{v}$ ) (JT-Baker Mexico) and $\mathrm{S}+\mathrm{F}$ received $30 \%$ commercially refined sucrose plus $10 \%$ fructose. The total energy intake received from water per day was: $\mathrm{C}: 0 \mathrm{~kJ} /$ day, 
S: $5.02 \mathrm{~kJ} /$ day, F: $1.67 \mathrm{~kJ} /$ day, and S + F: $6.69 \mathrm{~kJ} /$ day. The daily amount of food and liquid consumed was recorded, and food remaining from the previous day was replaced with a fresh supply. At the end of the experimental period, the rats were fasted for $12 \mathrm{~h}$ and then sacrificed by decapitation. Blood samples, visceral adipose tissue and hearts were obtained. Visceral adipose tissue was weighed to determine the adiposomatic index.

Blood biochemical analysis. Serum concentrations of glucose was determined using enzymatic colorimetric commercial diagnostic kits (Spinreact, Girona, Spain), and triglycerides (TG) and total cholesterol were determined by a colorimetric method (AccuLine plus kit using a Microlab 100, Merck, Dieren, The Netherlands), according to the manufacturer instructions.

Insulin resistance (IR). IR was evaluated by the homeostatic model assessment (HOMA-IR) and Quantitative Insulin Sensitivity Check Index (QUICKI). HOMA and QUICKI were calculated as follows: HOMA = fasting glucose $(\mathrm{nmol} / \mathrm{l}) \times$ fasting insulin $(\mu \mathrm{U} / \mathrm{l}) / 22.5$. QUICKI $=1 /(\log$ fasting insulin $(\mu \mathrm{U} / \mathrm{ml})+$ log fasting glucose $(\mathrm{mg} / \mathrm{dl})$. Serum insulin levels were measured by immuno-enzymatic assay (ELISA) using a commercial kit (Millipore, St Charles, MO, USA).

Arterial pressure (AP). AP was measured after the 12 weeks of treatment with a LE 5002 plethysmograph (Panlab, Harvard Apparatus USA), which uses the non-invasive "Tail Cuff" method. All animals were habituated for 3 days to reduce stress. Systolic arterial pressure (SAP), diastolic arterial pressure (DAP) and mean arterial pressure (MAP) were recorded. Data recording consisted of 3 to 4 repeated measurements per animal, and the average of those measurements was reported.

Western blot. Western blot analysis was performed in heart tissue to determine the relative expression of the atrial natriuretic peptide (ANP; as cardiac hypertrophy molecular marker), total CaMKII $\delta$ and ox$\mathrm{CaMKII}_{\delta}$. $50 \mathrm{mg}$ of macerated tissue was mixed with $1 \mathrm{ml}$ of RIPA buffer (50 mM HEPES, $150 \mathrm{mM} \mathrm{NaCl}, 1$ mM EDTA, $1 \%$ NP-40 and $25 \% \mathrm{C}_{24} \mathrm{H}_{39} \mathrm{NaO}_{4}$ ) supplemented with a cocktail of phosphatase and protease inhibitors $(2 \mu \mathrm{g} / \mathrm{ml}$ Aprotinine, $2 \mu \mathrm{g} / \mathrm{ml}$ Leupeptin, $1 \mu \mathrm{g} / \mathrm{ml}$ Pepstatin, $0.1 \mathrm{mM} \mathrm{PMSF}, 50 \mathrm{mM} \mathrm{NaF}$ and 1 $\mathrm{mM} \mathrm{Na}_{3} \mathrm{VO}_{4}$ ). Proteins were separated with $10 \%$ SDS-PAGE gels, transferred to polyvinylidene difluoride membranes, and then incubated with primary antibodies at the indicated dilution; 1:1000 for ANP (Santa

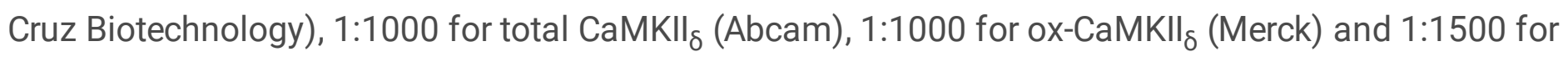
GAPDH (Santa Cruz Biotechnology). Once the solution with the primary antibody was removed, and membranes washed with T-TBS, they were incubated with the secondary antibody, Anti-Mouse 1:1000 for ANP and $\mathrm{CaMKII}_{\delta}$, and Anti-Rabbit 1:1000 for GAPDH and 1:3000 for ox-CaMKII ${ }_{\delta}$, for $2 \mathrm{~h}$ at room temperature. To detect the antibody signals, $1 \mathrm{ml}$ of Immobilon-Millipore Western HRP Substrate for chemiluminescent detection was added. Chemiluminescent proteins were revealed in a dark room using Kodak X-ray Plates, and bands intensity quantification was performed by densitometry using Image Studio Lite V. 5.2 (Li-Cor).

Lipid peroxidation assay and total nitrites assay. Assays were performed in the homogenized heart and serum samples as previously reported [19]. 
Superoxide dismutase (SOD). SOD activity was measured using a commercial kit (Sigma-Aldrich ${ }^{\circledR}$ kit No.1916). The heart and serum homogenate samples were incubated with the chromophore and enzyme, performing spectrophotometric measurements Tecan Infinite $200 \AA$ microplate reader at $425 \mathrm{~nm}$ every 30 $s$ for $5 \mathrm{~min}$ to estimate the activity of the enzyme present in the samples.

Histology. Hearts were fixed in $10 \%$ formalin, dehydrated, and embedded in paraffin for histological analysis. The tissues were cut in 7- $\mu \mathrm{m}$ slices, stained with hematoxylin and eosin and Masson's trichrome, and observed with an optical microscope to examine and evaluate any changes in the microarchitecture.

Cardiac hypertrophy determination. Gross determination of $\mathrm{CH}$ was obtained by measuring left and right ventricle wall thickness (RVWT and LVWT, respectively), the left ventricular cavity radius (LVCR), as well the left ventricular lumen area (LVLA) and the ratio between LVWT and LVCR (LVWT/LVCR). All the data images were analyzed using ImageJ 1.53j.

Statistical analysis. All the data were analyzed using GraphPad Prism 6.0 software. Data are presented as mean \pm SEM. To statistically compare experimental groups, analysis of variance (ANOVA) and a post hoc Tukey test were applied. $\mathrm{P}<0.05$ was considered significant.

\section{Results}

\section{Effect of high-sugar diets on body weight}

Table 1 shows that after 20 weeks of treatment, body weight (BW) showed no increase in any group of animals on the high-sugar diets compared to $C$. On the contrary, all BW values were below $C$, although only the decrease in $S(13 \%)$ was significant. On the other hand, food consumption was significantly lower in all experimental groups compared to $\mathrm{C}$, but liquid consumption was not significantly different among them and with C. However, visceral adipose tissue weight (VATW) and adiposomatic index (VATW/BW ratio) were significantly increased in all three hypercaloric groups compared to $\mathrm{C}$, yet the increase in these parameters were statistically higher in S $+\mathrm{F}$.

\section{Biochemical profile}

In relation to biochemical profile, glucose increased significantly with the 3 diets compared to C (by 45,24 and $51 \%$, for $S, F$ and $S+F$, respectively). The same trend was observed with insulin levels, which increased by $\sim 6$ - to $\sim 7$-fold in the 3 hypercaloric diets compared to $\mathrm{C}$. According to normal reference values for glucose and insulin, the carbohydrate-treated groups for 5 months could present DM2. The three experimental groups showed a clear IR caused by the hypercaloric diets, with values higher than the normal reference value of 3 , confirming the IR for HOMA, and values lower than the normal reference of 0.6 , confirming the IR for QUICKI. Regarding TG levels, these increased $\sim 2$-fold in S and F, while the increase was $\sim 3$-fold in $S+F$, compared to $C$. Total cholesterol increased significantly with all 3 diets 
compared to $C$ (by 16,12 and $34 \%$, for $S, F$ and $S+F$, respectively). For all cases, except for glucose, the $S$ $+\mathrm{F}$ group was statistically higher than the $\mathrm{S}$ and $\mathrm{F}$ groups (Table 2 ).

\section{Arterial pressure}

After 12 weeks of high carbohydrate intake, a significant increase in MAP values was observed in all experimental groups compared to $C$ (by 13,11 and $16 \%$ for, $S, F$ and $S+F$, respectively). SAP increased significantly (by $17 \%$ ) in $S+F$, whereas it was unchanged in groups $S$ and $F$. In relation to DAP, all 3 diet groups showed a significant increase compared to $C$ (by 17, 15 and 19\% for, $S, F$ and $S+F$, respectively). These results confirm that the $S$ and $F$ increased DAP, while $S+F$ caused an increase in SAP and DAP (Table 3).

\section{Oxidative stress}

MDA levels in cardiac tissue increased significantly ( $\geq 2$-fold) in animals fed with the three hypercaloric diets compared with $C$, although the increase was greater in $S+F(F i g .1 A)$. The same trend was found in serum MDA levels (Fig. 1B). Total nitrite levels in all three hypercaloric diets were significantly increased in both myocardium and serum compared with $\mathrm{C}$, although the increase was higher in animals treated with $F$ and $S+F$ than with $S$ (Fig. 1C, D). Regarding antioxidant activity, SOD was significantly decreased in $S, F$, and $S+F$ compared with $C$ in cardiac tissue (by 6,8 , and $13 \%$, respectively; Fig. $1 E$ ), but the decrease was higher in $S+F$. On the contrary, serum SOD activity was significantly increased in all three hypercaloric diets compared with C (by 21,19 , and $22 \%$, for S, F, and S + F, respectively; Fig. 1F).

Furthermore, a direct relationship was observed between the significant increase in VATW or TG and MDA, regardless of the type of sugar consumed. Except for the VATW-TG correlation in group C, all correlations were statistically significant. The higher the VATW, the higher the TG and MDA levels, therefore, there is a directly proportional relationship between these parameters (Fig. 2). In both cases, for VATW and TG, the correlation was higher in the $S+F$ group.

\section{Morphometric changes in the heart and histopathology.}

Although heart weight did not show significant differences between the $\mathrm{S}, \mathrm{F}$ and $\mathrm{S}+\mathrm{F}$ groups with respect to $C$, the left ventricular wall thickness (LVWT) increased significantly in all experimental groups compared to $C(50,44$ and $111 \%$ for $S, F$ and $S+F$, respectively). On the other hand, the right ventricular thickness (RVWT) also increased significantly in all three experimental groups compared to $C$ (by 55,63 and $104 \%$, in S, F and S + F, respectively) (Fig. 3 and Table 4). In relation to the left ventricular cavity radius (LVCR), it showed a significant decrease in the 3 diets $(65,50$ and $70 \%$, respectively). Finally, in the left ventricular lumen area (LVLA), the same trend was observed with a significant decrease in ventricular lumen after consumption of the diets (with a decrease of 72,64 and $80 \%$, respectively) (Fig. 3A and Table 4). According to these data (LVCR/LVWT), it can be suggested that concentric type hypertrophy developed in the 3 experimental groups, and that this was greater in the $S+F$ group. Furthermore, as Fig. 3B shows, there is an apparent increase in myocyte size and a concomitant increase in interstitial 
structure in the three diets compared to group C. Finally, Masson staining showed an increase in collagen deposition in the three diets compared to group C (Fig. 3C), which is a characteristic of pathological $\mathrm{CH}$.

\section{Western Blot}

The expression of ANP, a molecular marker of $\mathrm{CH}$, was significantly increased in all three groups of animals on hypercaloric diets, compared with $\mathrm{C}$. However, the increase was substantially greater in $\mathrm{S}+\mathrm{F}$ ( 4-fold increase), than in S and F ( $>$-fold increase in both groups; Fig. 4A). On the other hand, there was no change in total $\mathrm{CaMKII}_{\delta}$ in either experimental group (Fig. 3B). However, all hypercaloric diets significantly increased the ox-CaMKII $\delta$ levels (normalized to GAPDH and total CaMKII $\delta$, Fig. 4C and 4D, respectively), and this increase was higher in $S+F(\sim 2-, \sim 3-$, and $>3$-fold increase, in $S, F$, and $S+F$, respectively). This suggests that high sugar intake does not increase $\mathrm{CaMKII}_{\delta}$ expression in cardiac tissue, however, it does increase its pathological activation due to its oxidation by ROS.

\section{Discussion}

In this work we showed that chronic consumption of S and F, individually, lead to MetS, as indicated by the presence of IR and the increase in adiposomatic index and in the levels of glucose, insulin, totalcholesterol, TG, and ROS (as assessed by the levels of MDA and - $\mathrm{NO}_{2}$ ). Moreover, these metabolic alterations were accompanied by enhanced ox-CaMKII $\delta$ and by the development of $\mathrm{CH}$, suggesting that there is a relationship between the metabolic abnormalities produced by these sugars and the remodeling of cardiac structure. Finally, we showed that metabolic alterations as well as the ox-CaMKII $\delta$ and severity of $\mathrm{CH}$ are exacerbated when combined $\mathrm{S}$ and $\mathrm{F}(\mathrm{S}+\mathrm{F})$ are consumed.

Experimental studies have reported that high-carbohydrate diets produce metabolic alterations such as IR, glucose intolerance, OS, dyslipidemia, inflammation, increased AP, hepatic steatosis, among others [3, 8]. However, there are no experimental studies that compare the possible metabolic damage between the different sugars consumed from an early age and the cardiac alterations that they can generate. Therefore, in this study we evaluated the effects of long-term consumption of $S, F$ and $S+F$ in recently weaned Wistar rats.

BW in the three groups studied in this investigation did not increase; on the contrary, a significant reduction in weight was observed in the $S$ group. Our results are in agreement with previous studies, which showed that adult male Wistar rats fed with 30\% sucrose for 15-18 weeks, starting at a few weeks of age, did not present significant BW gain [3,20,21]. Nevertheless, despite the lack of BW gain, our results for the visceral adiposomatic index confirmed that animals on all three diets produced a greater accumulation of adipose tissue compared to $\mathrm{C}$, with the $\mathrm{S}+\mathrm{F}$ group inducing the greatest accumulation, followed by S, and finally F. These results indicate that the degree of fat accumulation depends on the type of sugar ingested. Our data agree with that reported by Fuente-Martin et al., who found that feeding neonatal Wistar rats with $33 \%$ sucrose for two months caused a significant decrease in BW, although there was a significant increase in adipose tissue due to adipocyte hypertrophy [22]. 
Although it is unclear why there was no BW gain in response to high-carbohydrate diets, it is likely due to the earlier age at which the animals started on the hypercaloric diets and the lower food intake they had, as seen in Table 1. These results are consistent with our previous reports showing that S-fed adults rats decreased food intake, while keeping constant liquid consumption [20-22]. Moreover, although it has been reported that rodent food provides a sufficient amount of protein and other essential nutrients, the preference of young animals for the intake of sugar-sweetened beverages $[21,22]$ and the decrease in solid food ingestion, may explain why there is not a BW gain in our animals despite the increase in visceral adipose tissue, as has been suggested by others [21, 22].

Biochemical profile showed that glucose, insulin, cholesterol and TG levels, as well as the HOMA index, increased significantly in $S$ and $F$ groups, which in addition to the increase in VATW and the decrease in QUICKY index, confirms that our animals developed several components of MetS, such as obesity, IR and dyslipidemia. Our data are in agreement with previous reports where the effects of S- and F-sweetened beverages were studied separately [3, 8, 22-24]. In this work, however, we also showed that combined $S$ and $\mathrm{F}$ diet exacerbates all these metabolic alterations, indicating that $\mathrm{S}+\mathrm{F}$ worsen MetS.

IR and sustained hyperglycemia promote increased proliferation of $\beta$-pancreatic cells to produce more insulin and compensate for excess blood glucose; however, they also induce the activation of other processes, such as inflammation and overproduction of ROS associated with mitochondrial oxidative stress [10]. According to our results, we confirmed that metabolic damage due to excess consumption of $\mathrm{S}$ and $\mathrm{F}$ as the main sugars used by the food industry, and especially when consumed together the metabolic damage is exacerbated. On the other hand, the data on hyperglycemia, hyperinsulinemia and IR suggest the development of DM2 in all experimental groups that consumed S, F or S + F. However, further tests are required to confirm the presence of DM2.

The consumption of high-carbohydrate diets produced a significant increase in both MAP and DAP, and only the S + F diet produced an increase in SAP. According to the WHO criteria, and taking into account that normal AP values in rats are similar to those in humans, our data show that hypercaloric diets increase AP. Balderas-Villalobos et al., reported that diet with sucrose (30\%) for 18 weeks did not cause a significant change in SAP and DAP, while Baños et al., reported that 3-week-old Wistar rats treated with the same diet for 8 months, resulted in a significant increase in SAP, but did not evaluate DAP [3, 25]. In this sense, the ENIGMA study examined a group of students with an average age of 20 years and with an elevated body mass index, and observed that DAP is the first parameter to rise and then give way to elevation of SAP or else, that only DAP was elevated [26]. This could explain why in our animals the significant increase in DAP was greater in the three diets. Nevertheless, in the combined intake $(S+F)$ the increase in both DAP and SAP (Table 3) may be due to exacerbation of OS (Fig. 1), which can lead to a greater endothelial damage (see below).

In the three groups of experimental animals, the intake of sugar-sweetened beverages significantly increased lipid peroxidation and, therefore, increased ROS generation (Fig. 1). Nonetheless, these effects were more accentuated with the S +F diet, suggesting a greater degree of OS. In the case of serum SOD, 
levels increased with all three hypercaloric diets, which could suggest that this enzyme is produced in higher amounts to maintain homeostasis between ROS and body antioxidants. However, this was not the case in cardiac tissue, where the sugary diets caused a reduction in SOD levels, probably due to the imbalance between antioxidant enzymes and increased ROS, characteristic of OS. Indeed, it has been reported that young adult Wistar rats (2 months old), placed on a $40 \%$ sucrose diet for 6 months, in addition to presenting obesity, IR and dyslipidemias, had a significant increase in hepatic MDA, and a significant decrease in SOD and glutathione, which are characteristic of OS in obesity [27].

The imbalance between ROS production and the activity of endogenous antioxidant agents in people with MetS has been strongly correlated with the development of different cardiovascular pathologies [5]. Zhang et al., reported that hearts from C57BL/6 mice (8 to 10 weeks old) treated with a 10\% F diet for 20 weeks, and neonatal rat ventricular cardiomyocytes cultured with $25 \mathrm{mM} \mathrm{F}$, displayed a significant increase in mitochondrial ROS [6]. Furthermore, echocardiographic and histological evaluation confirmed the presence of $\mathrm{CH}$ in these animals, which was accompanied by an increase in molecular markers such as ANP. These results highlighted that $\mathrm{F}$ by itself is able to induce an increase in mitochondrial ROS production, which is associated with the development of $\mathrm{CH}$, both in vivo and in vitro [6]. In this sense, our data showed that there is a direct correlation between VATW and the increase in TG and MDA (Fig. 2) and the severity of $\mathrm{CH}$ (Fig. 3 and Table 4) generated by the consumption of $\mathrm{S}$ and $\mathrm{F}$, thus, the higher the VATW, the higher the TG and OS and the higher $\mathrm{CH}$. Hence, combined consumption of $\mathrm{S}$ and $\mathrm{F}$, in addition to causing greatest VATW, generated highest OS and thus the highest $\mathrm{CH}$. On the other hand, it has been reported that presence of DM2, IR, glucose intolerance, inflammation and hepatic steatosis, besides obesity, plays an important role in the development of OS in MetS induced by high carbohydrate diets [5, 28]. Therefore, the higher levels in glucose, insulin and IR generated by $S+F$ diet, could explain why these sugars together caused higher MDA levels.

One of the mechanisms linking increased adipose tissue and $\mathrm{AH}$ is endothelial dysfunction, which is associated with decreased endothelial $\mathrm{NO}$ in blood vessels. As a result of excessive ROS production, $\mathrm{O}_{2}{ }^{--}$ is highly likely to react with NO leading to an increase in peroxynitrite (OONO--) [29]. This reaction is responsible for the decrease in NO, which together with the increase in OONO•-, contributes to endothelial dysfunction. Our data showed that hypercaloric diets produced an increase in - $\mathrm{NO}_{2}$ levels, which correlated with an increase in DAP in all groups. Nonetheless, the higher level of $-\mathrm{NO}_{2}$ produced by $\mathrm{S}+\mathrm{F}$ was accompanied by an increase not only in DAP, but also in SAP. Increase in both DAP and SAP correlates with higher $\mathrm{CH}$ when $\mathrm{S}$ and $\mathrm{F}$ are consumed in combination. A direct relationship in the development of $\mathrm{CH}$ by $\mathrm{AH}$-induced pressure overload has been widely reported [30]. It has been described that children with obesity, and with obesity plus $\mathrm{AH}$, had $\mathrm{CH}$, indicating that $\mathrm{CH}$ is not only present in adulthood, but also at early ages, and that its development is independently favored by AH and obesity [31]. These observations in children and adolescents support the hypothesis that from an early age obese subjects could develop $\mathrm{CH}$ or have a high probability of developing it in adulthood.

Increased expression of ANP has been established as a molecular marker of $\mathrm{CH}[13,32]$. Furthermore, it is well established that OS-mediated chronic activation of $\mathrm{CaMKII}_{\delta}$ plays an important role in the 
development of $\mathrm{CH}[6,13,17,18]$. Therefore, to determine the presence of $\mathrm{CH}$ in MetS and the molecular involvement of ox-CaMKII ${ }_{\delta}$, we evaluated the effects of hypercaloric diets on expression of ANP and level

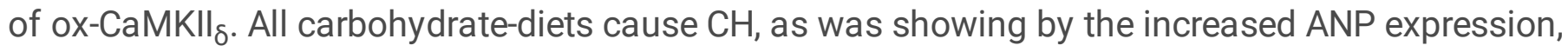
however, ANP expression was higher in S + F (Fig. 4A). In addition, expression of total CaMKII $\delta$ was not modified by any of the diets (Fig. 4B), which is in agree with a previous report [16], however, ox-CaMKII was increased by the three experimental groups, but this increase was higher in the case of $\mathrm{S}+\mathrm{F}$ (Fig. $4 \mathrm{C}$ and D). Recently, it was reported that $\mathrm{CaMKII}_{\delta}$ plays an important role in the development of obesityinduced $\mathrm{CH}$ [16]. Therefore, our results suggest that in MetS, combined $\mathrm{S}$ and $\mathrm{F}$ consumption exacerbates $\mathrm{CH}$ by increasing the level of ox-CaMKII $\delta$ (Fig. 5).

Histopathological analysis confirmed that all three diets, but mainly $\mathrm{S}+\mathrm{F}$ caused $\mathrm{CH}$ as indicated by increased LVWT and RVWT and decreased LVCR and LVLA (Table 4). These effects on cardiac structure decreased LVCR/LVWT index (Table 4), suggesting the development of a concentric-type $\mathrm{CH}$. Our histological studies also showed increased myocyte size and the presence of higher collagen deposition in the hearts of the three experimental groups (Fig. 3B and C). Increased myocyte size is usually accompanied by interstitial growth that allows structural support to $\mathrm{CH}$ [33], while collagen deposition takes place as a compensatory response to the cell loss caused mainly by apoptosis $[14,16]$. It has been reported that OS, produced under pathological conditions, plays a role in cardiomyocyte death and replacement of cell loss with collagen fibers [33]. In addition, OS has been implicated in the activation of $\mathrm{CaMKII}_{\delta}$ via oxidation of this kinase, which in turn, has been reported to contribute to cell growth and apoptosis in hearts [12-14]. Our data showing that the amount of VAT correlates with the level of OS (Fig. 2) and that $S+F$ causes the higher increase in these parameters and in parallel produces the higher increase in ox-CaMKII$\delta$ and $\mathrm{CH}$, suggest that there is a direct relationship between the degree of MetS and OS with the levels of ox-CaMKII $\delta$ and the worsening of $\mathrm{CH}$ (Fig. 5).

\section{Conclusions}

Our results demonstrate that excessive consumption of simple sugars, such as $S$ and $F$, from an early age leads to the development of metabolic disorders that lead to MetS and its complications such as $\mathrm{CH}$ and possibly DM2; however, these pathological conditions are aggravated when sugars are consumed in combination. On the other hand, we could prove that there is a direct relationship between MetS, OS, ox$\mathrm{CaMKII}_{\delta}$ and the worsening of $\mathrm{CH}$. This would be an alert to decrease the consumption of sugarsweetened beverages in children and adolescents to avoid severe metabolic damage and the development of $\mathrm{CH}$ and its complications in adulthood.

\section{Declarations}

\section{ACKNOWLEDGEMENTS}

The research was supported by CONACYT post-graduate fellowship no. 456311 . 


\section{DISCLOSURE STATEMENT}

The authors declare no conflict of interest.

\section{AUTHORS CONTRIBUTIONS}

G. Bravo and NL Gómez-Viquez contributed to the study design. DJ Arias-Chavez and P Mailloux-Salinas conceived experiments and analyzed data. DJ Arias-Chávez and J Altamirano contributed to helpful discussion and $\mathrm{F}$ Huang reviewed the manuscript.

The authors declare that all data were generated in-house and that no paper mill was used.

\section{DATA AVAILABILITY STATEMENT}

The authors confirm that the data supporting the findings of this study are available within the article.

\section{References}

1. Pepin A, Stanhope KL, Imbeault P (2019) Are fruit juices healthier than sugar-sweetened beverages? A review. Nutrients 11(5):1-16. https://doi.org/10.3390/nu11051006

2. Ng SW, Slining MM, Popkin BM (2012) Use of Caloric and Noncaloric Sweeteners in US Consumer Packaged Foods, 2005-2009. J Acad Nutr Diet 12(11):1828-1834.e6. https://doi.org/10.1016/j.jand.2012.07.009

3. Balderas-Villalobos J, Molina-Muñoz T, Mailloux-Salinas P, Bravo G, Carvajal K, Gómez-Viquez NL (2013) Oxidative stress in cardiomyocytes contributes to decreased SERCA2a activity in rats with metabolic syndrome. Am J Physiol - Hear Circ Physiol 305(9). https://doi.org/10.1152/ajpheart.00211.201

4. Feillet-Coudray C, Fouret G, Vigor C, Bonafos B, Jover B, Blachnio-Zabielska A et al (2019) Long-Term Measures of Dyslipidemia, Inflammation, and Oxidative Stress in Rats Fed a High-Fat/High-Fructose Diet. Lipids 54(1):81-97. https://doi.org/10.1002/lipd.12128

5. Szűcs G, Sója A, Péter M, Sárközy M, Bruszel B, Siska A et al (2019) Prediabetes Induced by FructoseEnriched Diet Influences Cardiac Lipidome and Proteome and Leads to Deterioration of Cardiac Function prior to the Development of Excessive Oxidative Stress and Cell Damage. Hindawi Oxidative Med Cell Longev 2019:3218275. https://doi.org/10.1155/2019/3218275

6. Zhang YB, Meng YH, Chang S, Zhang RY, Shi C (2016) High fructose causes cardiac hypertrophy via mitochondrial signaling pathway. Am J Transl Res 8(11). PMID: 27904687 PMCID: PMC5126329

7. Kitagawa A, Ohta Y, Ohashi K, Yashiro K, Fukuzawa K (2020) Effect of high fructose-induced metabolic syndrome on tissue vitamin e and lipid peroxide levels in rats. J Nutr Sci Vitaminol 66(2). https://doi.org/10.3177/jnsv.66.200

8. Moreno-Fernández S, Garcés-Rimón M, Vera G, Astier J, Landrier JF, Miguel M (2018) High fat/high glucose diet induces metabolic syndrome in an experimental rat model. Nutrients 10(10). 
https://doi.org/10.3390/nu10101502

9. Vona R, Gambardella L, Cittadini C, Straface E, Pietraforte D (2019) Biomarkers of oxidative stress in metabolic syndrome and associated diseases. Oxidative Medicine and Cellular Longevity 2019:8267234. https://doi.org/10.1155/2019/8267234

10. Aydin S, Aksoy A, Aydin S, Kalayci M, Yilmaz M, Kuloglu T et al (2014) Today's and yesterday's of pathophysiology: Biochemistry of metabolic syndrome and animal models. Nutrition 30(1):1-9. https://doi.org/10.1016/j.nut.2013.05.013

11. Erickson JR, Pereira L, Wang L, Han G, Ferguson A, Dao K et al (2013) Diabetic hyperglycaemia activates CaMKII and arrhythmias by O-linked glycosylation. Nature 502(7471). https://doi.org/10.1038/nature12537

12. Erickson JR, Joiner M, ling A, Guan X, Kutschke W, Yang J, Oddis CV et al (2008) A Dynamic Pathway for Calcium-Independent Activation of CaMKII by Methionine Oxidation. Cell 133(3). https://doi.org/10.1016/j.cell.2008.02.048

13. Erickson JR, Julie He B, Grumbach IM, Anderson ME (2011) CaMKII in the cardiovascular system: Sensing redox states. Physiol Rev 91(3):889-915. https://doi.org/10.1152/physrev.00018.2010

14. Feng N, Anderson ME (2017) CaMKII is a nodal signal for multiple programmed cell death pathways in heart. J Mol Cell Cardiol 103:102-109. http://dx.doi.org/10.1016/j.yjmcc.2016.12.007

15. Qu J, Mei Q, Niu R (2019) Oxidative CaMKII as a potential target for inflammatory disease (Review). Mol Med Rep 20(2):863-870. https://doi.org/10.3892/mmr.2019.10309

16. Zhong P, Quan D, Peng J, Xiong X, Liu Y, Kong B et al (2017) Role of CaMKII in free fatty acid/hyperlipidemia-induced cardiac remodeling both in vitro and in vivo. J Mol Cell Cardiol 109:116. https://doi.org/10.1016/j.yjmcc.2017.06.010

17. Mattiazzi A, Bassani RA, Escobar AL, Palomeque J, Valverde CA, Vila Petroff M et al (2015) Chasing cardiac physiology and pathology down the caMKII cascade. Am J Physiol-Hear Circ Physiol 308(10):H1177-H1191. https://doi.org/10.1152/ajpheart.00007.2015

18. Federico M, Portiansky EL, Sommese L, Alvarado FJ, Blanco PG, Zanuzzi CN et al (2017) Calciumcalmodulin-dependent protein kinase mediates the intracellular signalling pathways of cardiac apoptosis in mice with impaired glucose tolerance. J Physiol 595(12). https://doi.org/10.1113/JP273714

19. Colado-Velázquez J, Mailloux-Salinas P, Medina-Contreras J, Cruz-Robles D, Bravo G (2015) Effect of Serenoa Repens on oxidative stress, inflammatory and growth factors in obese wistar rats with benign prostatic hyperplasia. Phyther Res (10):1525-1531. https://doi.org/10.1002/ptr.5406

20. Carvajal K, El Hafidi M, Marin-Hernández A, Moreno-Sánchez R (2005) Structural and functional changes in heart mitochondria from sucrose-fed hypertriglyceridemic rats. Biochim Biophys Acta Bioenerg 1709(3). https://doi.org/10.1016/j.bbabio.2005.08.001

21. El Hafidi M, Cuéllar A, Ramírez J, Baos G (2001) Effect of sucrose addition to drinking water, that induces hypertension in the rats, on liver microsomal $\Delta 9$ and $\Delta 5$-desaturase activities. $J$ Nutr Biochem 12:396-403. https://doi.org/10.1016/S0955-2863(01)00154-1 
22. Fuente-Martín E, García-Cáceres C, Granado M, Sánchez-Garrido MA, Tena-Sempere M, Frago LM et al (2012) Early postnatal overnutrition increases adipose tissue accrual in response to a sucroseenriched diet. Am J Physiol - Endocrinol Metab 302(12).

https://doi.org/10.1152/ajpendo.00618.2011

23. Kim M, Do GY, Kim I (2020) Activation of the renin-angiotensin system in high fructose-induced metabolic syndrome. Korean J Physiol Pharmacol 24(4).

https://doi.org/10.4196/KJPP.2020.24.4.319

24. Johnson RJ, Segal MS, Sautin Y, Nakagawa T, Feig DI, Kang DH et al (2007) Potential role of sugar (fructose) in the epidemic of hypertension, obesity and the metabolic syndrome, diabetes, kidney disease, and cardiovascular disease. Am J Clin Nutr 86(4):899-906. https://doi.org/10.1093/ajcn/86.4.899

25. Baños G, Medina-Campos ON, Maldonado PD, Zamora J, Pérez I, Pavón N et al (2005) Activities of antioxidant enzymes in two stages of pathology development in sucrose-fed rats. Can J Physiol Pharmacol 83(3). https://doi.org/10.1139/y05-013

26. McEniery CM, Yasmin, Wallace S, Maki-Petaja K, McDonnell B, Sharman JE et al (2005) Increased stroke volume and aortic stiffness contribute to isolated systolic hypertension in young adults. Hypertension 46(1). https://doi.org/10.1161/01.HYP.0000165310.84801.e0

27. Souza Cruz EM, Bitencourt de Morais JM, Dalto da Rosa CV, da Silva Simões M, Comar JF, de Almeida Chuffa LG et al (2020) Long-term sucrose solution consumption causes metabolic alterations and affects hepatic oxidative stress in Wistar rats. Biol Open 9(3):bio047282. https://doi.org/10.1242/bio.047282

28. Sulzbacher Da Silva B, Macedo A, Paulino B, Taffarel M, Borba IG, Ortega Telles L et al (2021) High sucrose diet attenuates oxidative stress, inflammation and liver injury in thioacetamide-induced liver cirrhosis. Life Sci 267:118944. https://doi.org/10.1016/j.Ifs.2020.118944

29. Martínez-abundis E, Sánchez-rodríguez MA, Hafidi-bentlakder E (2005) Participación de la mitocondria en el desarrollo de estrés oxidativo en la obesidad Asociación Mexicana de Bioquímica Clínica, AC. Bioquimia (30): 82-89. Available from:

https://www.medigraphic.com/pdfs/bioquimia/bq-2005/bq053d.pdf

30. Wang C-H, Pandey S, Sivalingam K, Asokan Shibu M, Kuo W-W, Padma Viswanadha V et al (2021) Leech extract: A candidate cardioprotective against hypertension-induced cardiac hypertrophy and fibrosis. J Ethnopharmacol 264:113346. https://doi.org/10.1016/j.jep.2020.113346

31. Brady TM (2016) The Role of Obesity in the Development of Left Ventricular Hypertrophy Among Children and Adolescents. Curr Hypertens Rep 18(1):3. https://doi.org/10.1007/s11906-015-0608-3

32. Cerrudo CS, Fermepin MR, Cavallero S, Saucedo SL, Hertig CM, Fernández BE (2011) Natriuretic peptides synthesis and secretion profiles during the evolution of cardiac hypertrophy in doca-salt hypertensive rats. Rev Argent Cardiol 79(6). https://doi.org/10.7775/rac.79.6.725

33. Shimizu I, Minamino T (2016) Physiological and pathological cardiac hypertrophy. Journal of Molecular Cellular Cardiology Academic Press 97:245 - 62. 
https://doi.org/10.1016/j.yjmcc.2016.06.001

\section{Tables}

Table 1 Body weight

\section{Experimental Groups}

\begin{tabular}{lllll} 
Parameter & $\mathrm{C}$ & $\mathrm{S}$ & $\mathrm{F}$ & $\mathrm{S}+\mathrm{F}$ \\
\hline BW $(\mathrm{g})$ & $514.3 \pm 10.2$ & $448.3 \pm 20.9^{*}$ & $501.3 \pm 21.5^{\mathrm{a}}$ & $470.5 \pm 16.4$ \\
\hline Food consumption $(\mathrm{g})$ & $19.9 \pm 0.2$ & $7.68 \pm 0.2^{*}$ & $7.77 \pm 0.2^{*}$ & $7.64 \pm 0.5^{\star}$ \\
\hline Liquid consumption $(\mathrm{mL})$ & $42.83 \pm 1.22$ & $40.83 \pm 0.90$ & $42.50 \pm 0.86$ & $40.17 \pm 1.31$ \\
\hline VATW $(\mathrm{g})$ & $7.04 \pm 0.49$ & $14.75 \pm 1.14^{*}$ & $12.32 \pm 0.73^{*}$ & $16.13 \pm 1.29^{*}, \mathrm{~b}$ \\
$\begin{array}{l}\text { Adiposomatic Index \% } \\
\text { (VATW/BW) }\end{array}$ & $1.37 \pm 0.09$ & $3.13 \pm 0.20^{*}$ & $2.48 \pm 0.13^{*, \mathrm{a}}$ & $3.80 \pm 0.30^{*, \mathrm{a} . \mathrm{b}}$ \\
\hline
\end{tabular}

Values represent mean \pm s.e.m. ANOVA one way. Post hoc Tukey. ${ }^{*} p<0.05$ vs Control. ${ }^{a} p<0.05$ vs Sucrose Group. ${ }^{b} p<0.05$ vs Fructose Group. C: Control, S: Sucrose, F: Fructose and S + F: Sucrose + Fructose. BW: Body Weight VATW: Visceral Adipose Tissue Weight. $\mathrm{n}=12$.

Table 2 Biochemical parameters

\section{Experimental Groups}

\begin{tabular}{|c|c|c|c|c|}
\hline Biochemical parameters & C & $S$ & $\mathrm{~F}$ & $S+F$ \\
\hline $\begin{array}{l}\text { Glucose } \\
(\mathrm{mg} / \mathrm{dl})\end{array}$ & $93.7 \pm 2.8$ & $135.5 \pm 3.9 *$ & $116.6 \pm 2.7^{*, a}$ & $141.8 \pm 2.8^{*, b}$ \\
\hline $\begin{array}{l}\text { Insulin } \\
(\mu \mathrm{g} / \mathrm{dl})\end{array}$ & $9.3 \pm 0.3$ & $64.9 \pm 1.7^{*}$ & $53.7 \pm 1.1^{*, \mathrm{a}}$ & $70.8 \pm 1.6^{* a, b}$ \\
\hline RI-HOMA & $2.3 \pm 0.1$ & $20.6 \pm 0.7^{*}$ & $14.9 \pm 0.6^{*, a}$ & $25.6 \pm 0.3^{*}, \mathrm{a}, \mathrm{b}$ \\
\hline RI-QUICKI & $0.58 \pm 0.008$ & $0.37 \pm 0.002^{*}$ & $0.39 \pm 0.003^{*, a}$ & $0.36 \pm 0.001 *, b$ \\
\hline $\begin{array}{l}\mathrm{TG} \\
(\mathrm{mg} / \mathrm{dl})\end{array}$ & $69.8 \pm 3.2$ & $132.4 \pm 6.4^{\star}$ & $144.9 \pm 4.2^{*}$ & $202.8 \pm 9.5^{\star}, \mathrm{a}, \mathrm{b}$ \\
\hline Total Cholesterol (mg/dl) & $69.5 \pm 1.5$ & $80.3 \pm 2.5^{\star}$ & $77.9 \pm 1.1^{\star}$ & $93 \pm 1.6^{*}, \mathrm{a}, \mathrm{b}$ \\
\hline
\end{tabular}


Table 3. Arterial pressure

\section{Experimental Groups}

\begin{tabular}{lllll}
$\begin{array}{l}\text { Arterial Pressure } \\
(\mathrm{mm} \mathrm{Hg})\end{array}$ & $\mathrm{C}$ & $\mathrm{S}$ & $\mathrm{F}$ & $\mathrm{S}+\mathrm{F}$ \\
\hline MAP & $100.6 \pm 2.5$ & $113.2 \pm 1.9^{*}$ & $111.5 \pm 2.8^{*}$ & $116.8 \pm 1.9^{*}$ \\
\hline SAP & $122.3 \pm 0.8$ & $129.7 \pm 1.9$ & $128.6 \pm 2.8$ & $143.5 \pm 2.0^{*}$, a, b \\
\hline DAP & $89.9 \pm 3.9$ & $105 \pm 2.0^{*}$ & $103.6 \pm 2.8^{*}$ & $106.8 \pm 4.0^{*}$
\end{tabular}

Values represent mean \pm s.e.m. ANOVA one way. Post hoc Tukey. ${ }^{\star} p<0.05$ vs Control. ${ }^{a} p<0.05$ vs Sucrose Group. ${ }^{b} p<0.05$ vs Fructose Group. C: Control, S: Sucrose, F: Fructose and S + F: Sucrose + Fructose. SAP: systolic arterial pressure. DAP: diastolic arterial pressure. MAP: Mean arterial pressure. $\mathrm{n}=12$.

Table 4 Heart morphometric data

\begin{tabular}{|lllll|}
\hline \multicolumn{5}{|c|}{ Experimental Groups } \\
\hline Parameters & $\mathrm{C}$ & $\mathrm{S}$ & $\mathrm{F}$ & $\mathrm{S}+\mathrm{F}$ \\
\hline $\mathrm{HW}(\mathrm{g})$ & $1.818 \pm 0.022$ & $1.809 \pm 0.056$ & $1.827 \pm 0.030$ & $1.823 \pm 0.028$ \\
\hline $\begin{array}{l}\mathrm{LVWT} \\
(\mathrm{mm})\end{array}$ & $2.325 \pm 0.048$ & $3.500 \pm 0.147^{*}$ & $3.350 \pm 0.065^{*}$ & $4.925 \pm 0.137^{*}, \mathrm{a}, \mathrm{b}$ \\
\hline $\begin{array}{l}\text { RVWT } \\
(\mathrm{mm})\end{array}$ & $1.075 \pm 0.047$ & $1.675 \pm 0.075^{*}$ & $1.775 \pm 0.063^{*}$ & $2.200 \pm 0.057^{*}, \mathrm{a}, \mathrm{b}$ \\
\hline $\begin{array}{l}\text { LVCR } \\
(\mathrm{mm})\end{array}$ & $2.700 \pm 0.074$ & $0.925 \pm 0.052^{*}$ & $1.450 \pm 0.020^{*}$ & $0.800 \pm 0.054^{*}, \mathrm{a}, \mathrm{b}$ \\
\hline $\begin{array}{l}\text { LVLA } \\
\left(\mathrm{mm}{ }^{2}\right)\end{array}$ & $1.830 \pm 0.025$ & $0.503 \pm 0.075^{*}$ & $0.655 \pm 0.041^{*}, \mathrm{a}, \mathrm{b}$ & $0.358 \pm 0.020^{*}, \mathrm{a}, \mathrm{b}$ \\
\hline LVCR/LVWT & $1.164 \pm 0.053$ & $0.264 \pm 0.006^{*}$ & $0.433 \pm 0.011^{*}, \mathrm{a}, \mathrm{b}$ & $0.163 \pm 0.013^{*}, \mathrm{a}, \mathrm{b}$ \\
\hline
\end{tabular}

Values represent mean \pm s.e.m. ANOVA one way. Post hoc Tukey. ${ }^{*} p<0.05$ vs Control. ${ }^{a} p<0.05$ vs Sucrose Group. ${ }^{b} p<0.05$ vs Fructose Group. C: Control- white bars, S: Sucrose-orange bars, F: Fructoseblue bars and S+F: Sucrose + Fructose- green bars. $n=4$. HW: Heart Weight. LVWT: Left Ventricular Wall Thickness. RVWT: Right Ventricular Wall Thickness. LVRC: Left Ventricular Cavity Radius.

\section{Figures}



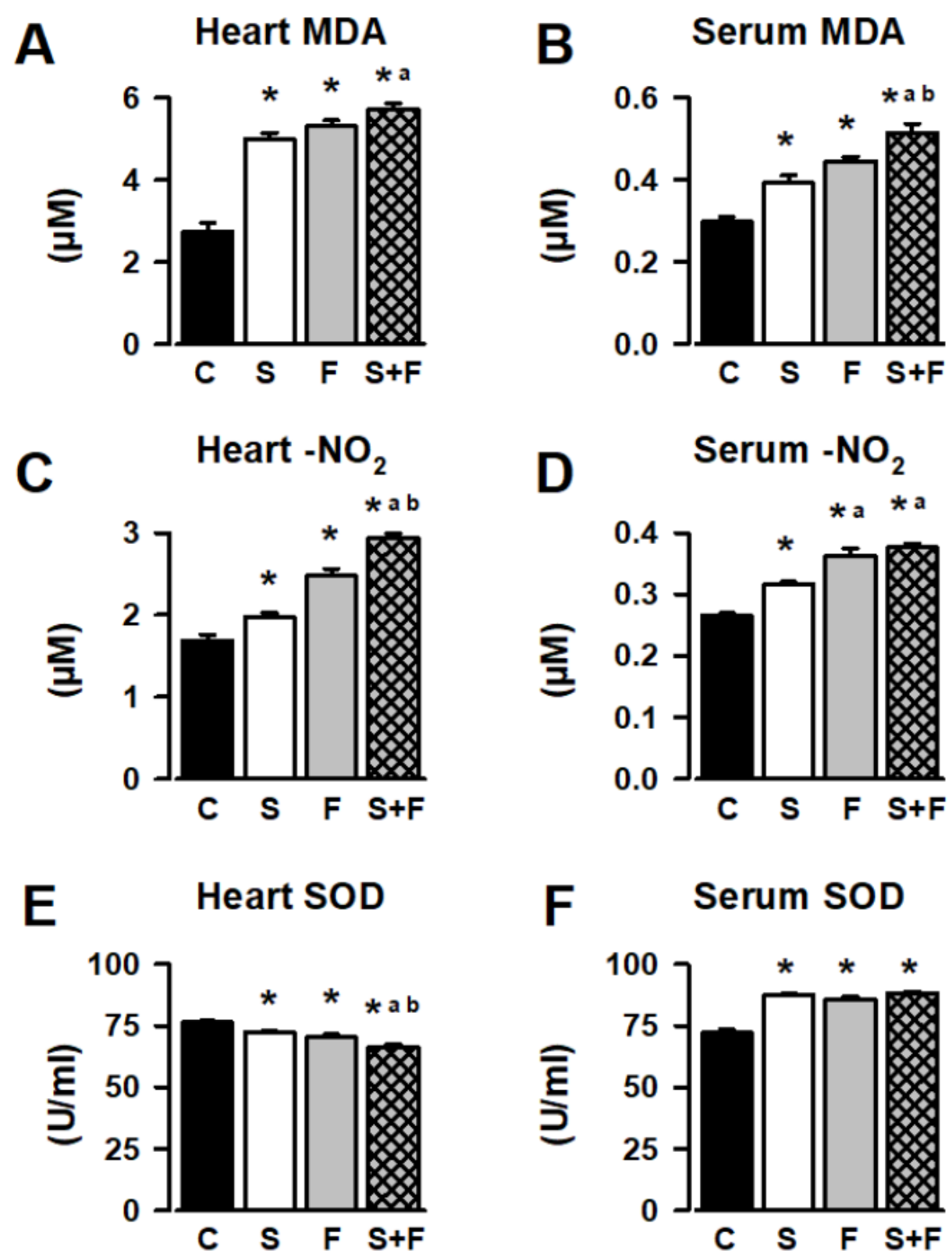

Figure 1

Oxidative Stress. A. MDA in heart. B. MDA in serum. C. -NO2 in heart and D. -NO2 in serum. E. SOD in heart, F. SOD in serum. Values represented as mean \pm s.e.m. ANOVA one way. Post hoc Tukey. ${ }^{*} \mathrm{p}<0.05$ vs Control. ap $<0.05$ vs Sucrose Group. bp $<0.05$ vs Fructose Group. MDA: Malondialdehyde. -NO2: Total nitrites. SOD: Superoxide Dismutase. $\mathrm{n}=8$. 


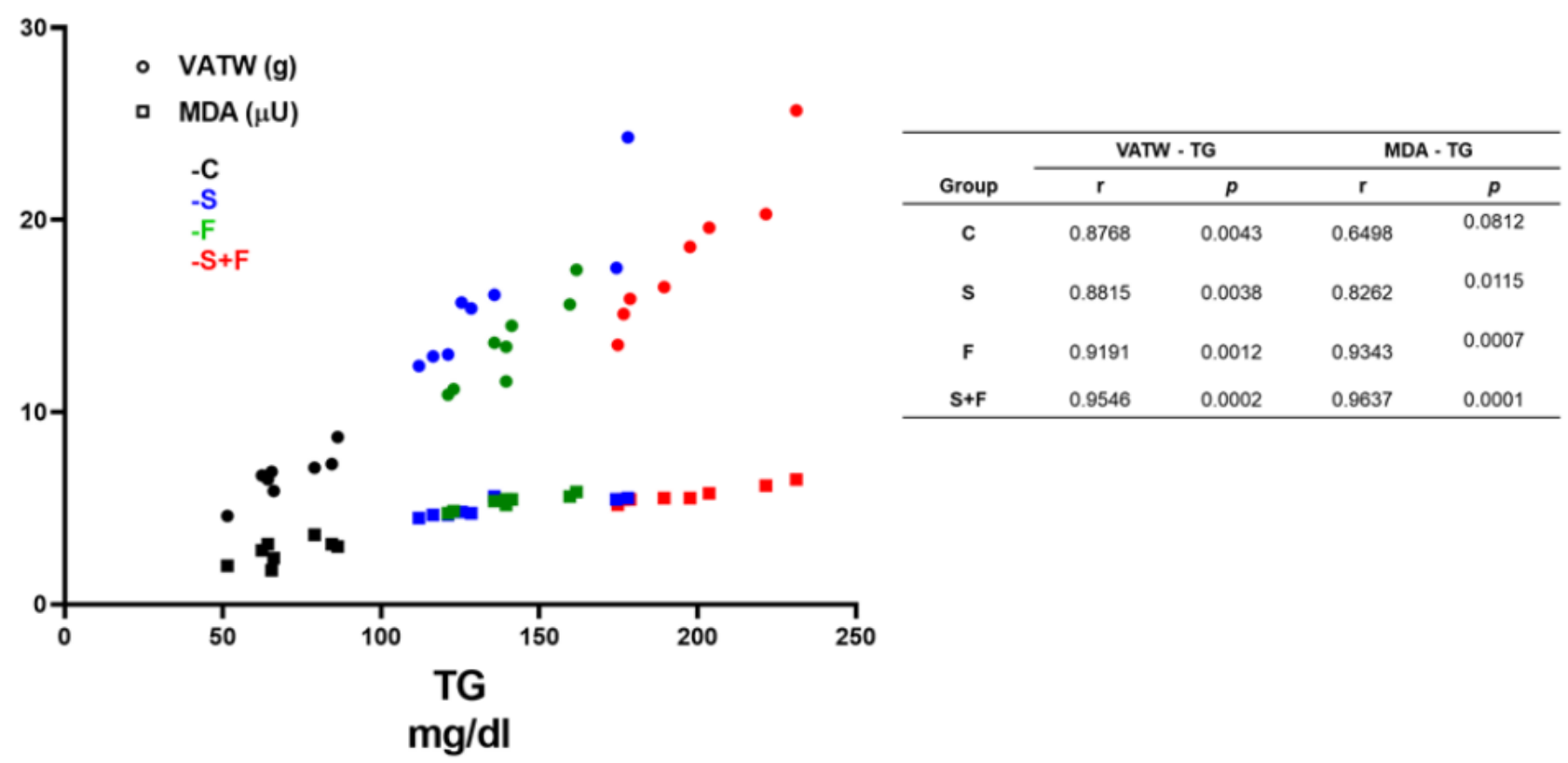

Figure 2

Correlations between visceral adipose tissue, triglycerides and malondialdehyde. Adipose tissue was positively correlated with serum triglyceride and malondialdehyde levels in all groups. Values represented as mean \pm s.e.m. Correlations were performed with Pearson's $r$. ${ }^{*} p<0.05$. S: Sucrose, F: Fructose, S+F: Sucrose + Fructose.TG: triglycerides. Values VATW: visceral adipose tissue weight. MDA: Malondialdehyde. 

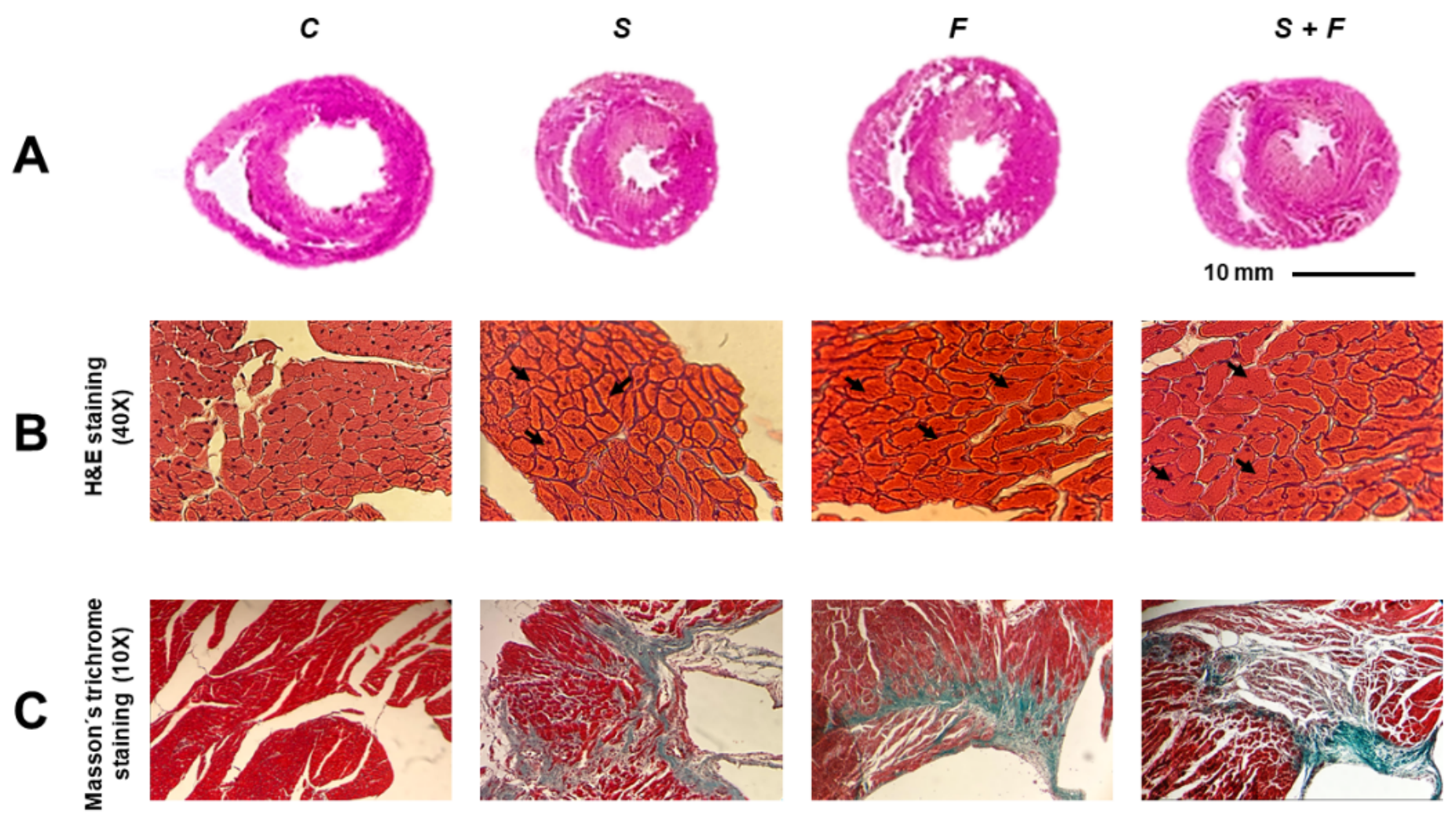

\section{Figure 3}

Histopathological staining of hearts. A: Cross section of hearts with H\&E staining. B. Cardiac cells of the left ventricular wall with H\&E staining. C. Cardiac cells of the left ventricular wall with Masson's trichrome C: Control, S: Sucrose, F: Fructose, S+F: Sucrose + Fructose. H\&E: Hematoxylin-Eosin. Viewed at 40X magnification for each slice. $\downarrow$ Hypertrophied cardiomyocytes. 
A
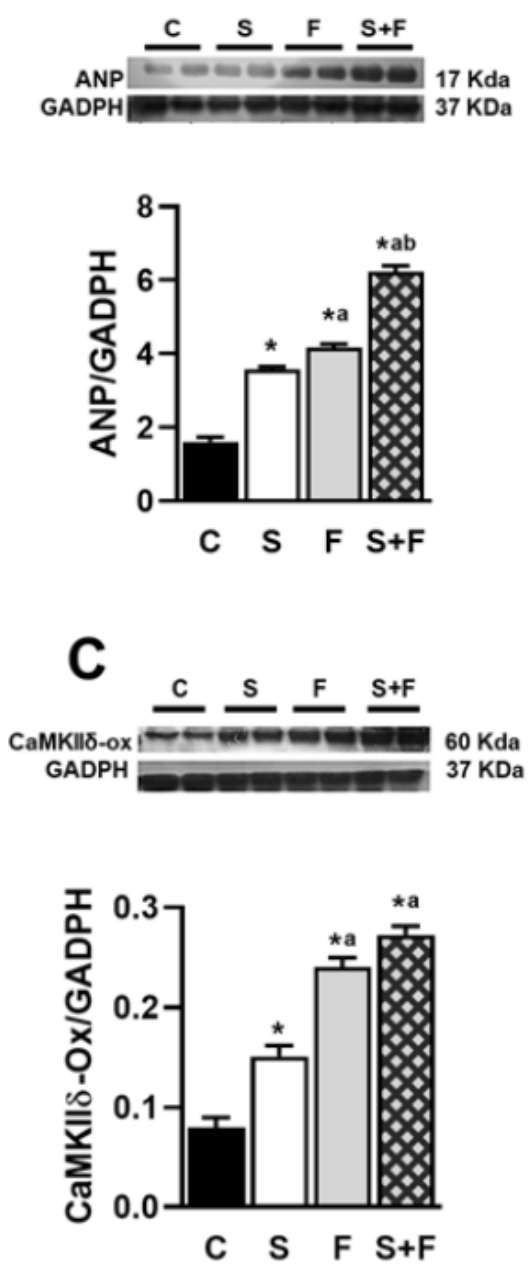

B
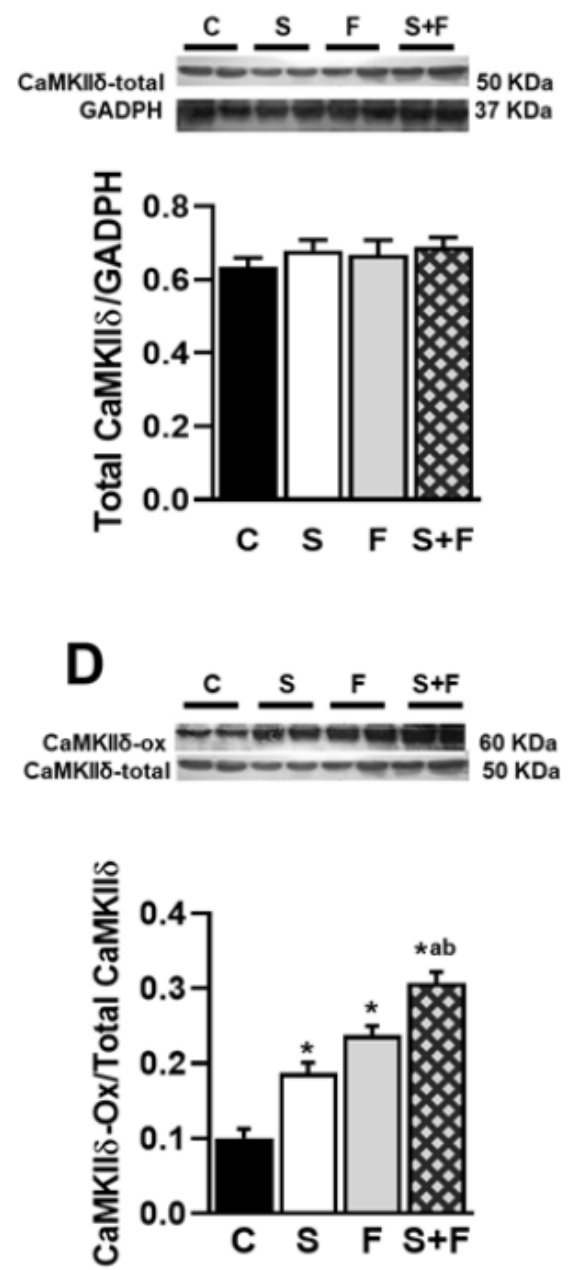

Figure 4

Immunoblots and Western Blot analysis of cardiac hypertrophy. A. ANP expression. B. CaMKIIס-total expression. C. ox-CaMKIII density. D. Relation ox-CaMKIII/ CaMKIII-total. Values represented as mean \pm s.e.m. ANOVA one way. Post hoc Tukey. ${ }^{*} p<0.05$ vs Control. ap $<0.05$ vs Sucrose Group. bp $<0.05$ vs Fructose Group. n=4. ANP: Atrial Natriuretic Peptide. 


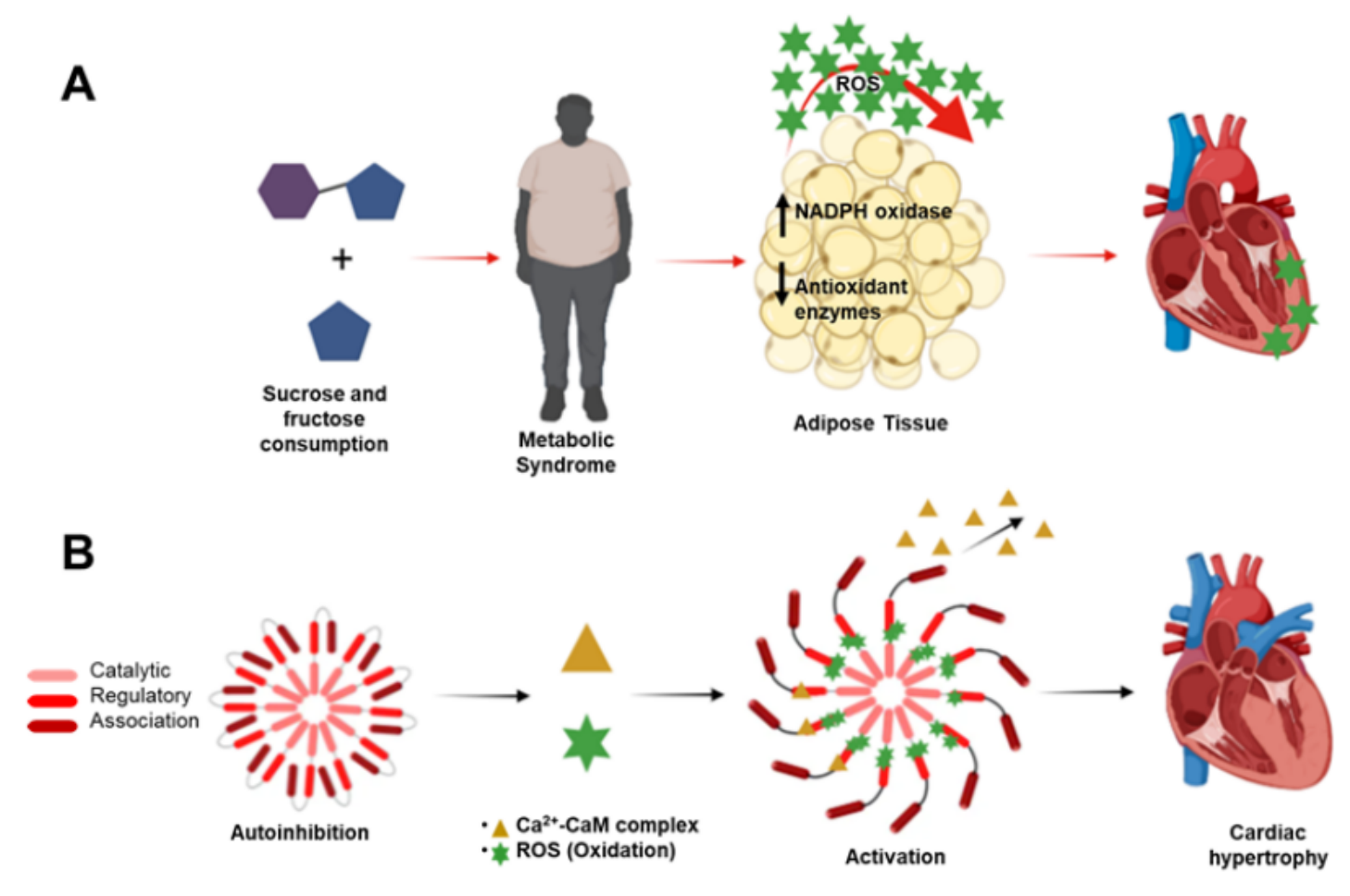

\section{Figure 5}

Possible generation of cardiac hypertrophy in metabolic syndrome. A: The consumption of sucrose and fructose in excess generates metabolic syndrome characterized by the presence of obesity and excess adipose tissue. This adipose tissue is an important source of reactive oxygen species causing oxidative stress in different organs such as the heart. B. CaMKIII activation is carried out by Ca2+-CAM complex binding to the regulatory domain, however, when this binding is reversed CaMKII $\delta$ is autoinhibited. However, $\mathrm{Ca} 2+-\mathrm{CAM}$ complex-independent activation also occurs. Oxidation at the regulatory site of methionine 281 and 282 is a type of Ca2+-CAM-independent activation of this enzyme, moreover, it is dependent on the production of reactive oxygen species and a state of oxidative stress. This activation is known as pathological activation because it leads to the development of different abnormal conditions such as cardiac hypertrophy. 